\title{
Perceived quality of physiotherapist-led orthopaedic triage compared with standard practice in primary care: a randomised controlled trial
}

Karin S. Samsson ${ }^{1,2^{*}}$, Susanne Bernhardsson ${ }^{3}$ and Maria E. H. Larsson ${ }^{1,3}$

\begin{abstract}
Background: Physiotherapist-led orthopaedic triage, where physiotherapists diagnose and determine management plans, aims to enhance effectiveness and provide the best care. However, scientific evidence for the effectiveness of this model of care remains limited, and there are few studies reporting on patients' perceptions of the care provided.

The purpose of this study was to evaluate patients' perceived quality of care in a physiotherapist-led orthopaedic triage in primary care, compared with standard practice.
\end{abstract}

Methods: In a randomised controlled trial, patients of working age referred for orthopaedic consultation at a primary healthcare clinic in Sweden received either physiotherapist-led triage $(n=102)$ or standard practice (orthopaedic surgeon assessment) ( $n=101)$. Neither subjects nor clinicians were blinded.

The questionnaire Quality from the Patient's Perspective (QPP) was used to evaluate perceived quality of care focusing on the caregivers' medical-technical competence and identity-orientated approach. Also, to what extent patients' expectations were met, and their intention to follow advice was evaluated.

Results: For this study, 163 patients (80\%) were analysed (physiotherapist-led triage $(n=83)$, standard practice $(n=80))$. Participants perceived significantly higher quality of care with the triage than with the standard practice in regards to receiving best possible examination and treatment (medical-technical competence) $(p<0.001)$. This was also found in regards to receiving information about examination and treatment $(p<0.001)$, results $(p<0.001)$, and self-care $(p<0.001)$, the caregiver's understanding $(p<0.001)$, respect $(p<0.001)$ and commitment $(p<0.001)$ as well as the opportunity to participate in decision-making $(p=0.01)$ (identity-orientated approach). Participants in the physiotherapist-led triage group reported to a significantly higher extent that their expectations of the treatment were met $(p<0.001)$, as well as the intent to follow the advice and instructions received $(p=0.019)$.

(Continued on next page)

\footnotetext{
* Correspondence: karin.samsson@vgregion.se

${ }^{1}$ Department of Health and Rehabilitation, Institute of Neuroscience and

Physiology at Sahlgrenska Academy, University of Gothenburg, Box 430405

30 Gothenburg, Sweden

${ }^{2}$ Närhälsan Tjörn Rehabilitation Clinic, Primary Health Care, Region Västra

Götaland, Syster Ebbas väg 1, 47194 Kållekärr, Sweden

Full list of author information is available at the end of the article
} 
(Continued from previous page)

Conclusions: This paper reports on patients' perceptions of quality of care in a physiotherapist-led orthopaedic triage compared with standard practice. Patients in both groups reported that they perceived good quality of care, with the patients in the physiotherapist-led triage reporting significantly higher perceived quality of care than those in the standard practice group. This model of care seems to meet patients' expectations and result in a greater intention to follow advice and instructions for self-management.

Our findings are in line with existing literature that this model of care provides an opportunity to shape patient-centered care that can improve access and offer care on the most appropriate level, with maintained good quality of care.

Trial registration: Clinical Trials NCT02265172. Registered 10 June 2014

Keywords: Advanced practice physiotherapy, Physical therapy, Quality of care, Patient perception, Sweden, Expectations

\section{Background}

Considering the increasing demand on health care to provide accurate and timely diagnosis and care for patients with musculoskeletal complaints, alternative models of care, such as physiotherapist-led orthopaedic triage, have been explored predominantly in the UK, Australia and Canada [1-10]. The aims of triage have been suggested to be to reduce long waiting times and to enhance effectiveness and best care/practice, i.e. timely access to the right care from the appropriately qualified health care professionals who can direct patients towards the optimal treatment pathway [11]. Physiotherapists have been used to triage, diagnose, and determine management plans, and to refer for investigations, orthopaedic surgery or conservative management $[3,12,13]$. Studies of this model of care, in various outpatient settings, have reported high agreement on diagnosis and treatment approach between physiotherapists and orthopaedic surgeons $[4,8,10,14,15]$. Several studies have shown that physiotherapist-led orthopaedic triage also decreases referrals for orthopaedic consultation $[1,5,15-17]$. Also, emerging evidence suggests that such a model of care can improve access to care with equal or better outcomes compared to standard practice (i.e. orthopaedic surgeon consultation) regarding waiting times, treatment effectiveness, use of healthcare resources, economic costs, both patient and provider satisfaction, and patient outcomes such as pain and functional disability $[18,19]$.

We have previously reported primary outcomes from a randomised controlled trial investigating physiotherapist-led orthopaedic triage in a Swedish primary healthcare setting [20]. The study showed significantly better selection accuracy and shorter waiting times with physiotherapist-led triage than with standard practice. Furthermore, the study showed no differences between physiotherapy-led triage and standard practice regarding the secondary outcome long-term follow-up of patient-reported outcome measures [21]. In this paper, we report on the secondary outcome patients' perceived quality of care with this model of care.

Several studies have reported that patients are either equally $[3,6]$ or even more satisfied with physiotherapist-led triage than with standard practice $[4,8]$. Still, scientific evidence for the effectiveness of this model of care remains limited and there is a scarcity of high-quality studies [22]. Few of the studies report on health outcomes using standardised outcome measures [11]. Additionally, considering differences among national healthcare systems, studies of such a model need to be conducted in each respective country [23].

Good care should be patient-centred [24], which is characterised by respectful and individualised care. One of the core components of patient-centred care is participation in clinical decision-making, which empowers patients to actively engage in their care [25]. Therefore, patients' perceptions of the quality of their health care are of increasing interest for healthcare policy makers $[24,26]$. Additionally, evaluating the patients' perceptions is essential to any new role involving a shift in traditional practice boundaries [27].

Wilde et. al. [28] have presented a model that stipulates that patients' perceptions of what constitutes quality of care are formed by their encounters with an existing care structure, and by their norms, expectations, and experience. Based on this model, the questionnaire Quality from the Patient's Perspective (QPP) was developed.

Considering that in many countries it is still standard practice for patients to be assessed by orthopaedic surgeons when referred for orthopaedic consultation, we were interested in exploring patients' perceptions of the quality of care of a physiotherapist-led triage. Even though previously reported results indicate that physiotherapist-led orthopaedic triage could provide appropriate care for the patients, patients' perceptions of this model need to be investigated before large scale 
implementation. Therefore, the purpose of this paper was to evaluate patients' perceived quality of care in a physiotherapist-led orthopaedic triage in primary care compared with standard practice. Furthermore, we wanted to evaluate outcome-related aspects: whether patients' expectations were met, and patients' intention to follow advice and instructions.

\section{Methods}

\section{Study design}

This paper reports findings from a randomised controlled trial, and the full study design and method of this trial have been reported previously [20, 21]. The hypothesis of the trial was that this new model of care would provide good selection accuracy, without negatively affecting patient-related outcomes and while maintaining good quality of care. From this trial, two papers have been previously published: one reported on the primary outcome selection accuracy for orthopaedic intervention [20], and one reported long-term effects on patientreported outcomes [21].

\section{Setting and participants}

The study took place at a primary healthcare centre in a Swedish municipality. Consecutive recruitment of patients referred for orthopaedic surgeon consultation at the healthcare centre was performed between August 2009 and January 2011 with the following inclusion criteria: working age (between 18 and 67 years of age), subacute (four weeks to three months) or persistent (>three months) musculoskeletal pain, and the ability to understand written and spoken Swedish. The exclusion criteria were chosen in collaboration with the orthopaedic surgeon in the study. Patients were excluded if the stated diagnosis on the referral was hallux valgus, ganglion or trigger finger, where the general practitioners (GPs) were assumed to have high accuracy in diagnosis.

\section{Procedure}

We used a block randomisation with a 1:1 allocation and block sizes of 20 to ensure an equal allocation ratio [29]. Sealed, opaque envelope containing details of the allocated group were mixed and put in a box by an administrator. After receiving verbal consent for participation, the administrator randomised the patient by drawing the next envelope from the box. Due to the nature of the intervention it was not possible to blind therapists or participants to their group allocation.

\section{Physiotherapist-led orthopaedic triage}

The physiotherapist in this trial, also the first author of this paper, did not receive any training specific for this trial. She had specialist training in the form of postgraduate qualifications, including a master's degree in
Manipulative Therapy, one year of mentored clinical practice within the scope of orthopaedic manual therapy (OMT) and eight years of clinical experience in primary care, four within the scope of OMT. The duration of the appointment was up to 60 min with the main aims to diagnose and determine the most appropriate management pathway. The patients also received a brief treatment comprised of advice on ergonomics and/or exercises when needed; however, only during the one visit. Management pathways consisted of one or more of the following referrals; further investigation (i.e. $x$-rays, MRI), orthopaedic surgeon consultation (i.e. appropriate candidate for surgery), physiotherapy or occupational therapy (for conservative management with on-going support) or back to the patient's GP. If patients were found to be appropriate candidates for surgery, the physiotherapist had the authority to make an appointment with the orthopaedic surgeon at the healthcare centre, without consideration of the waiting list. This was made to ensure that if the patients were considered appropriate for surgery, the total waiting time for consultation with the orthopaedic surgeon was not longer than the patients in the standard practice group due to their participation in the study. Referrals for further investigations were requested and sent via the patient's GP and the images could be assessed together with the orthopaedic surgeon, if needed. One or two optional follow-up visits were offered when needed, for example follow-up after treatment or investigations.

\section{Standard practice}

The orthopaedic surgeon in this trial had 26 years of experience in orthopaedic medicine, 21 of which were as an orthopaedic specialist. The duration of the appointment was $15 \mathrm{~min}$, with the main aims to diagnose and determine the most appropriate management pathway. The patients received advice, prescriptions or injections, when needed. Management pathways were the same as for the triage group with the addition of orthopaedic intervention (i.e. minor surgery at the present healthcare centre), and referral to orthopaedic clinic for orthopaedic intervention (i.e. appropriate candidates for surgery). One or two optional follow-up visits were offered when needed, for example follow-up after investigations.

\section{Outcome measures}

Patients' perceptions of quality of care were assessed using the QPP. As per the choice of the participant, the questionnaire was sent either by post or as an online survey approximately five days after the appointment. Up to two reminders were sent. To reduce risk of bias, distribution and administration of the questionnaires were handled by an independent administrative company, ImproveIT (Halmstad, Sweden). The QPP is a self- 
administered questionnaire developed using a grounded theory approach and consisting of items formulated in words used by patients [28]. The questionnaire has been psychometrically tested $[30,31]$ and validated in different settings [32-34]. A short version of the questionnaire has been psychometrically tested [35-38]. The short, swedish version was used in this study, with minor modifications to include the physiotherapist and the orthopaedic surgeon. The QPP questionnaire is based on the assumption that patients' perception of quality of care may be considered in four dimensions: caregivers' medical-technical competence; care organisations' physical-technical conditions; degree of identityorientation in the caregivers' attitudes and actions; and the care organisations' socio-cultural atmosphere [32]. The primary outcomes reported in this paper were the following two dimensions (comprising eight items): medical-technical competence (one item) and identity-orientated approach (seven items regarding information, participation, and the caregiver's commitment, understanding and respect). Each item is evaluated in two ways using a 4-point Likert scale; first the patients rate how they perceive the quality of care (PR; perceived reality);"This is what I experienced...", ranging from 1 (Do not agree at all) to 4 (Completely agree). The patients then also rate how important that aspect of care is (SI; subjective importance);"This is how important it was to me...", ranging from 1 (Little or no importance) to 4 (Of the very highest importance). Each item also has a "Not applicable" response option. The secondary outcomes were two additional items from the QPP measuring outcome-related aspects: "Will you follow the advice and instructions that you have now received from the physiotherapist/ orthopaedic surgeon?" (response options rated on a 3point Likert scale ranging from 1 (No) to 3 (Yes, completely), or Not applicable (Don't know or I have not received any advice or instructions)), and "To what extent were your expectations of the treatment met?" rated on a 5-point Likert scale ranging from 1 (Not at all) to 5 (To a very large extent).

Demographic data including age, sex, civil status, country of birth, and education were collected to describe the study population.

\section{Sample size}

Sample size was originally calculated based on the trial's main outcome variable, selection accuracy, as previously reported [20]. To verify that the study had sufficient power to detect a difference in the secondary outcome, QPP, a retrospective power calculation was made. This was based on QPP mean scores for the item"I received the best possible examination and treatment (as far as I can tell)" (range 1-4). A relevant mean difference between groups for items from the QPP has been suggested to be 0.35 [39]. Analysis were made using an online calculator from University of British Columbia, Canada [40].

\section{Data analysis}

Descriptive statistics for demographic characteristics were used and analysed with the Independent $t$-test or the Pearson's Chi-squared test to determine any baseline differences between the groups. Between-group comparisons were made for the QPP data using the MannWhitney $U$-test, and medians, quartile 1 and 3 , and means are reported. The significance level was set to $p<0.05$.

Demographic data of responders vs. non-responders were analysed within each respective group using the Independent $t$-test and the Pearson's Chi-squared test.

The QPP data were registered by Improve IT staff with the KUPPIT ${ }^{1}$ software (ImproveIT, Halmstad, Sweden, 2003). All collected data were transferred and analysed by the first author using IBM SPSS, version 18.0 (IBM Corp, Armonk, NY, USA).

\section{Results \\ Participants}

The inclusion and analysis process is illustrated in Fig. 1. The total response rate for the QPP was $80 \%$ (81\% in the physiotherapist-led triage group and $79 \%$ in the standard practice group). The reasons for non-response are unknown. There were no significant baseline differences between the two groups with respect to sex, civil status, and country of birth, education or occupation; however, participants in the standard practice group were significantly older (Table 1 ).

\section{Study power}

In this study, the mean difference for the item"I received the best possible examination and treatment (as far as I can tell)" (measured on a 1-4 scale), was 0.60 units (triage group mean 3.51 vs. standard practice group mean 2.91, SD 0.94), and with a sample size of 73 participants in each group (respondents to this particular item) we reached sufficient power $(0.97)$ to detect differences at the $p<0.05$ level.

\section{Quality of care from the patient perspective}

As presented in Table 2 participants perceived significantly higher quality of care with the triage assessment than with the orthopaedic surgeon assessment; both regarding receiving best possible examination and treatment (medical-technical competence) and regarding receiving information, the opportunity to participate in decision making, and the caregiver's understanding, respect and commitment (identity-orientated approach). Perceived importance of the different items was rated 


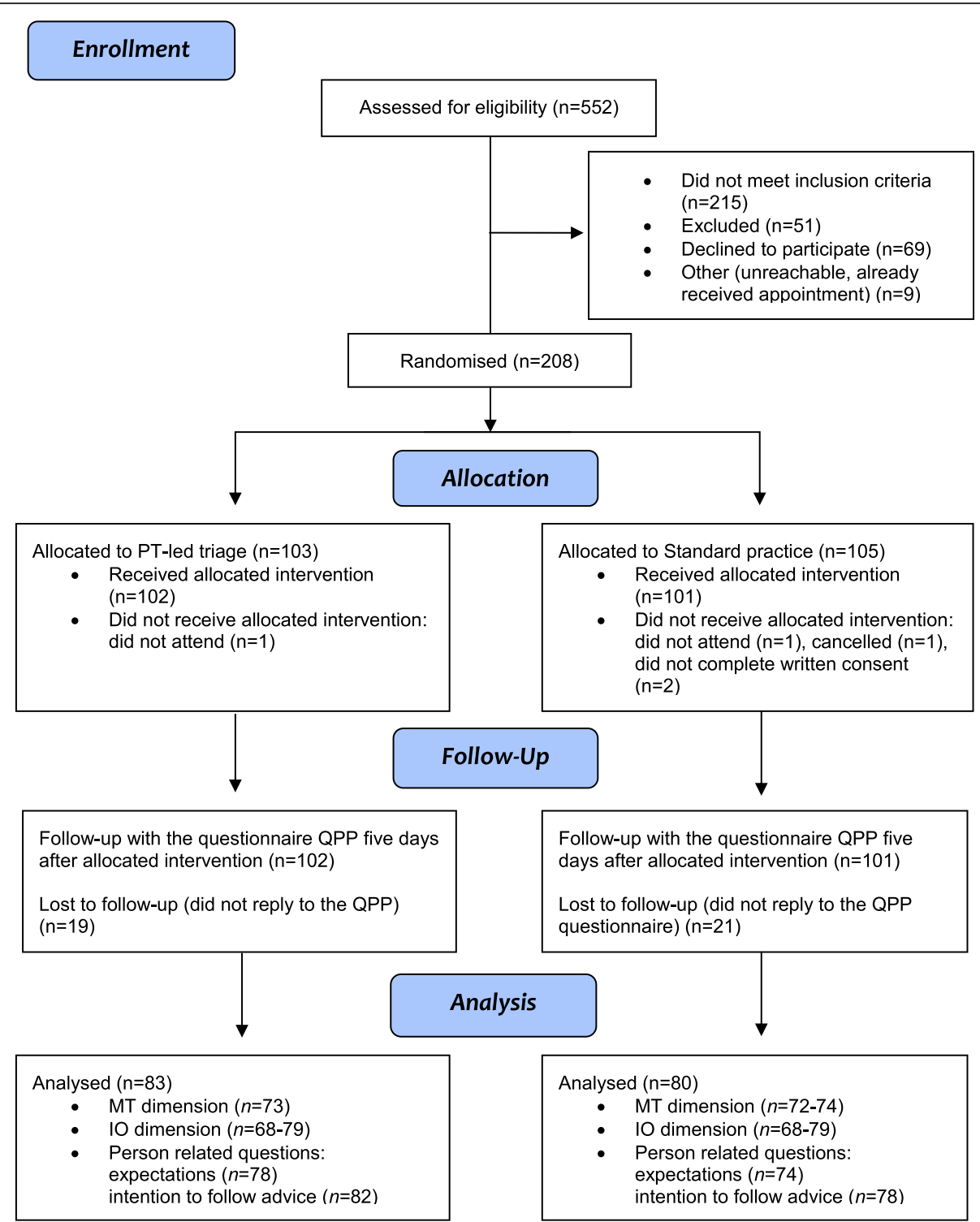

Fig. 1 CONSORT flow chart of the progress of participants through the study. PT = physiotherapist, QPP = Quality from the patients' perspective, MT = Medical-technical competence, $\mathrm{IO}=$ Identity-orientated approach

higher by patients in the triage group for the item in the Medical-technical competence dimension and for two of the items in the Identity-orientated approach dimension.

\section{Outcome-related aspects}

Participants in the physiotherapist-led triage group reported to a significantly higher extent that their expectations of the treatment were met, as well as the intent to follow the advice and instructions received, when compared with the standard practice group (Table 3).

\section{Missing data analysis}

The missing data analyses showed significant demographic differences between those who responded to the
QPP and those who did not. There was a significant difference within both groups: those who did not respond were born outside of Sweden to a higher extent (triage $p=0.004$, standard practice $p=0.02$ ). Furthermore, there were significant differences in the standard practice group: those who did not respond were younger $(p=$ $0.001)$, lived alone $(p=0.007)$, and had a lower education level $(p=0.04)$ than those who responded.

\section{Discussion}

This paper reports on patients' perceptions of quality of care in a physiotherapist-led orthopaedic triage compared with standard practice. Patients in both groups reported that they perceived good quality of care, with the 
Table 1 Demographic characteristics of the respondents of the QPP questionnaire at baseline

\begin{tabular}{|c|c|c|}
\hline & $\begin{array}{c}\text { Physiotherapist-led } \\
\text { triage } n=83 \\
\mathrm{n}(\%)\end{array}$ & $\begin{array}{c}\text { Standard practice } \\
n=80 \\
\mathrm{n}(\%)\end{array}$ \\
\hline \multicolumn{3}{|l|}{ Age (years) ${ }^{*}$} \\
\hline Mean & 51 & 55 \\
\hline Range & $18-67$ & $21-67$ \\
\hline SD & 10.6 & 12.5 \\
\hline \multicolumn{3}{|l|}{ Sex } \\
\hline Male & $38(46)$ & $36(45)$ \\
\hline Female & $45(54)$ & $44(55)$ \\
\hline \multicolumn{3}{|l|}{ Civil status } \\
\hline Married/living together & $69(83)$ & $73(91)$ \\
\hline Single/living alone & $14(17)$ & $7(9)$ \\
\hline \multicolumn{3}{|l|}{ Country of birth } \\
\hline Sweden & $81(98)$ & $76(95)$ \\
\hline Other & $2(2)$ & $4(5)$ \\
\hline \multicolumn{3}{|l|}{ Education } \\
\hline Elementary school & $10(12)$ & $19(24)$ \\
\hline Upper secondary school & $38(46)$ & $33(41)$ \\
\hline University & $35(42)$ & $28(35)$ \\
\hline \multicolumn{3}{|l|}{ Occupation } \\
\hline Working & $61(73)$ & $53(67)$ \\
\hline Student & $2(2)$ & $2(2)$ \\
\hline Other & $20(24)$ & $25(31)$ \\
\hline
\end{tabular}

QPP Quality from the Patients' Perspective, $S D$ Standard Deviation

"Statistically significant difference between groups $(p=0.036)$

patients in the physiotherapist-led triage group reporting that they perceived significantly higher quality of care. This model of care seems to meet patients' expectations and result in a greater intention to follow advice and instructions for self-management.

\section{Quality of care from the patient perspective}

The findings in this paper are in line with previous studies reporting higher patient satisfaction with care and services received after a physiotherapist-led triage [4, 8]. We found that the patients in the triage group reported perceiving a higher medical-technical competence of the caregiver, similar to the findings of Razmjou et al. [8]. They also found significantly higher patient satisfaction regarding technical skills after physiotherapy triage compared with standard practice in a speciality shoulder clinic. Reeve et al [41] found in their qualitative study of patients' perspectives of quality of a physiotherapist's spinal screening service, that patients expect clinicians to be appropriately qualified and skilled.

It has previously been reported that patients expect and want information about the whole process of care, as well as provision of a diagnosis and instructions for self-management [41, 42]. The patients in the physiotherapist-led triage group in our study reported to a higher extent that they received adequate information about the whole care process. Razmjou et al. [8], also found significant differences in favour of the physiotherapist regarding explanation of the results and answers to questions. An important part of physiotherapy is education and information about symptoms and how they relate to potential underlying conditions. and it has been reported that patients attending a physiotherapist-led triage even felt less pain and anxiety upon receiving information about their problem [3]. Patients have previously reported that the most important expectation when consulting a clinician (physiotherapist or GP) is not to recover but to have their disorder confirmed [42, 43].

The patients in the physiotherapist-led triage group reported to a higher extent that the clinicians seemed to understand, respect, commit and care about them, also this in concordance with previously reported findings [8].

The results of this study suggest that this model of care is patient-centred. A narrative review and synthesis of the literature by Kitson et al. [25] identifies three core elements of patient-centred care: patient participation and involvement, relationship between the patient and the healthcare professional, and the context in which care is delivered. The authors conclude that all health professionals provide care based on these elements, but to a varying degree depending on the interest and priority given to these elements by the professional group. There is a possibility that the two different professions (physiotherapists and orthopaedic surgeons) have different responsibilities and therefore also different focus of care.

The opportunity to participate in the clinical decisionmaking was rated higher by the patients in the physiotherapist-led triage group. This is one of the core concepts of patient-centred care and several other studies have reported a desire of patients with musculoskeletal disorders to participate in the clinical decision-making process and feel involved in the process of the consultation [41, 42]. This could be achieved by good communication and information regarding the whole triage process. May et al. [44] found that failure to communicate effectively about the condition and the treatment options was the most frequent source of patient dissatisfaction amongst patients with back pain. It could be argued that since the primary outcome for an orthopaedic consultation is to decide whether the patient is suitable for surgery or not, there is not much room for participation. However, patients are generally less satisfied than surgeons with surgery outcomes, and one of the explanations for this is a difference in expectations of outcomes [45]. It has therefore been recommended that patients are 
Table 2 Participants' perceptions of the quality of care in the dimensions of medical-technical competence and identity-oriented approach

\begin{tabular}{|c|c|c|c|c|c|c|c|c|c|c|c|c|}
\hline \multirow[t]{2}{*}{ Dimension/factor } & & \multicolumn{6}{|c|}{ Physiotherapist-led triage $n=83$} & \multicolumn{5}{|c|}{ Standard practice $n=80$} \\
\hline & & $n$ & NA & Median & Q1;Q3 & Mean & $n$ & NA & Median & Q1;Q3 & Mean & $p$-value \\
\hline \multicolumn{13}{|l|}{ Medical-technical competence } \\
\hline \multicolumn{13}{|l|}{ Care received } \\
\hline \multirow[t]{2}{*}{ I received the best possible examination and treatment. } & PR & 73 & 2 & 4 & $3 ; 4$ & 3.5 & 74 & 4 & 3 & $2 ; 4$ & 2.9 & $<0.001$ \\
\hline & $\mathrm{SI}$ & 73 & 2 & 4 & $3 ; 4$ & 3.5 & 72 & 5 & 3 & $3 ; 4$ & 3.3 & 0.022 \\
\hline \multicolumn{13}{|l|}{ Identity-oriented approach } \\
\hline \multicolumn{13}{|l|}{ Receiving information about } \\
\hline \multirow[t]{2}{*}{ How examinations and treatments would take place. } & PR & 79 & 1 & 4 & $3 ; 4$ & 3.6 & 69 & 5 & 3 & $2 ; 4$ & 3.0 & $<0.001$ \\
\hline & $\mathrm{SI}$ & 79 & 1 & 3 & $3 ; 4$ & 3.1 & 67 & 6 & 3 & $3 ; 4$ & 3.0 & 0.559 \\
\hline \multirow[t]{2}{*}{ The results of examinations and treatments. } & PR & 70 & 5 & 4 & $3 ; 4$ & 3.4 & 68 & 1 & 3 & $2 ; 4$ & 2.9 & $<0.001$ \\
\hline & $\mathrm{SI}$ & 71 & 4 & 3 & $3 ; 4$ & 3.3 & 65 & 3 & 3 & $3 ; 4$ & 3.1 & 0.166 \\
\hline \multirow[t]{2}{*}{ Self-care: "how I should take care of myself". } & PR & 69 & 4 & 4 & $3 ; 4$ & 3.4 & 61 & 7 & 3 & $2 ; 4$ & 2.7 & $<0.001$ \\
\hline & $\mathrm{SI}$ & 68 & 5 & 3.5 & $3 ; 4$ & 3.3 & 59 & 8 & 3 & $3 ; 4$ & 3.1 & 0.159 \\
\hline \multicolumn{13}{|l|}{ Participation in decision making } \\
\hline \multirow[t]{2}{*}{ I had opportunity to participate in decisions. } & $P R$ & 74 & 4 & 4 & $3 ; 4$ & 3.6 & 68 & 8 & 3.5 & $3 ; 4$ & 3.2 & 0.010 \\
\hline & $\mathrm{SI}$ & 74 & 4 & 4 & $3 ; 4$ & 3.5 & 67 & 8 & 4 & $3 ; 4$ & 3.3 & 0.227 \\
\hline \multicolumn{13}{|l|}{ Caregiver's understanding, respect, and commitment } \\
\hline \multirow[t]{2}{*}{ Seemed to understand how I experienced my situation. } & PR & 77 & 0 & 4 & $4 ; 4$ & 3.8 & 73 & 1 & 3 & $2.5 ; 4$ & 3.1 & $<0.001$ \\
\hline & $\mathrm{SI}$ & 77 & 0 & 4 & $3 ; 4$ & 3.6 & 71 & 2 & 4 & $3 ; 4$ & 3.4 & 0.046 \\
\hline \multirow[t]{2}{*}{ Was respectful towards me. } & PR & 74 & 0 & 4 & $4 ; 4$ & 3.9 & 72 & 1 & 4 & $3 ; 4$ & 3.4 & $<0.001$ \\
\hline & $\mathrm{SI}$ & 74 & 0 & 4 & $3 ; 4$ & 3.7 & 70 & 2 & 4 & $3 ; 4$ & 3.5 & 0.090 \\
\hline \multirow[t]{2}{*}{ Showed commitment: cared about me. } & PR & 76 & 0 & 4 & $4 ; 4$ & 3.9 & 69 & 1 & 3 & $2 ; 4$ & 3.0 & $<0.001$ \\
\hline & $\mathrm{SI}$ & 76 & 0 & 4 & $3.25 ; 4$ & 3.7 & 67 & 2 & 4 & $3 ; 4$ & 3.5 & 0.039 \\
\hline
\end{tabular}

Results from the questionnaire Quality from the Patients' Perspective (QPP). Item scores for PR (Perceived Reality) ranged from 1 (do not agree at all) to 4 (completely agree) and for SI (Subjective Importance) from 1 (little or no importance) to 4 (of the very highest importance). NA: Not applicable. Q1; Q3: First quartile; third quartile. Statistically significant differences between groups (two-tailed $p$-value) are presented in bold font

extensively informed and to a higher extent participate in decision making [45].

Interestingly, the patients in the physiotherapist-led triage group reported to a higher extent that it was important to receive the best possible care, and that it was important that the caregiver was committed and understanding. It could be that expectations of physiotherapists differ from those of an orthopaedic surgeon; however, no studies addressing this issue have been found. Still, it has previously been reported that patients demand different quality of care depending on the care situation itself [46]. As previously mentioned, the focus of the different aspects of patient-centred care tends to vary with the professional group [25] and it could be that the expectations of patients also vary.

\section{Outcome-related aspects}

The patients in the physiotherapist-led triage group reported to a higher extent that their expectations were met and that they were more inclined to follow the advice from the physiotherapist, which could be correlated to their care experience [28]. A clear association has previously been established between patient experience and self-rated and objectively measured health outcomes,

Table 3 Outcome-related aspects of quality of care; meeting of expectations and intentions to follow advice and instructions

\begin{tabular}{|c|c|c|c|c|c|c|c|c|c|c|c|}
\hline & \multicolumn{6}{|c|}{ Physiotherapist-led triage $n=83$} & \multicolumn{5}{|c|}{ Standard practice $n=80$} \\
\hline & $n$ & NA & Median & Q1;Q3 & Mean & $n$ & NA & Median & Q1;Q3 & Mean & $p$-value \\
\hline Meeting of expectations & 78 & 0 & 4 & $4 ; 5$ & 4.3 & 74 & 0 & 4 & $3 ; 4$ & 3.7 & $<0.001$ \\
\hline Intention to follow advice and instructions & 76 & 6 & 3 & $3 ; 3$ & 2.8 & 59 & 19 & 3 & $2 ; 3$ & 2.6 & 0.019 \\
\hline
\end{tabular}

Results from the questionnaire Quality from the Patients' Perspective (QPP). Response options ranged from 1 (not at all) to 5 (to a very large extent) for the item regarding expectations and from 1 (no) to 3 (yes, completely) for the item regarding intentions. Q1; Q3: First quartile; third quartile, NA: Not applicable. Statistically significant differences between groups (two-tailed $\mathrm{p}$-value) are presented in bold font 
such as adherence to recommended medication and treatments as well as use of healthcare resources [47]. Also, greater patient treatment satisfaction has been found to be associated with better adherence as well as improved persistence to recommendations or treatments [47, 48].

The finding in this study that patients in the physiotherapist-led triage group reported to a higher extent that they received useful advice on treatment and self-care, is consistent with previous research which has shown that information, exercise and pain relief are part of the physiotherapist assessment to a larger extent than assessments by other medical staff $[4,5,49,50]$. It has been found that patients expect and wish for training programmes and advice about self-management, when seeking health care for back and neck pain [42, 43]. Considering that a large number of patients referred for orthopaedic consultation are managed non-surgically, this could be important for the patients' wellbeing as well as further care-seeking.

\section{Methodological considerations}

Not many studies of quality of care have used a randomised controlled design. Together with the large sample size, sufficient power and the study's originality, this is a major strength. Furthermore, a validated satisfaction questionnaire which addresses patients' perceptions of different aspects of care was used. The total response rate for the QPP $(80 \%)$ can be considered high, as a review of patient satisfaction with health care reported a mean response rate of $67 \%$ for postal questionnaires [51].

Nevertheless, this study also has several limitations. There is a potential risk of performance bias in the analysis and interpretation of data since the first author was the physiotherapist performing the triage in this study, as well as responsible for the main part of the data analysis and writing of this paper. However, she was not involved in the eligibility assessment, randomisation or data collection, and all the data were coded during analysis. It could seem that the physiotherapist had a strong stake in the outcome of the study and therefore performed beyond usual care; however, the orthopaedic surgeon and all other healthcare personnel involved were briefed on the study protocol, and therefore one can argue that everyone had similar stakes and performed at their best.

The choice to use block randomisation was made to enable a balanced distribution of patients between the groups. Each block consisted of 20 envelopes and although it is a large block size which makes it harder to detect allocation sequence, there is a risk of selection bias.

Each patient consulted one of the clinicians only, which limits the possibility of investigating inter-rater agreement. Preferably, the protocol should consist of several physiotherapists and orthopaedic surgeons and also, by both healthcare professionals assessing the same patients. Due to the clinical reality at the present healthcare centre, with only one physiotherapist with appropriate level of knowledge as well as only one orthopaedic surgeon, such a protocol was not feasible. Also, on national level, many primary healthcare centres are small, and very few have orthopaedic surgeons or physiotherapists with advanced knowledge. There is a possibility that the interpersonal attributes of the therapist have influenced the outcome more than the professional roles [52]. Studies have shown that patient satisfaction with care is more related to interactions with the therapist and attributes of the therapist, than to treatment outcome $[52,53]$. Due to the nature of the intervention neither the care providers nor the participants could be blinded, which might have affected the outcome.

The duration of the assessment was set according to standard practice at many clinics at the time of the study, and therefore differed between the groups (15 versus $60 \mathrm{~min}$ ). This was done to mirror clinical practice and avoid disruptions, and to facilitate future implementation. Considering that previous research has found that having adequate time can be a determinant of satisfaction [52], the longer duration of the physiotherapist-led triage could have affected the outcome $[4,8]$.

Considering the minor change to the QPP questionnaire to include the physiotherapist or orthopaedic surgeon this is not likely to have affected reliability and validity of the questionnaire. Since the QPP was administrated by the Improve IT staff, the risk for detection bias is low. There is a potential risk of attrition bias due to significant demographic differences between those who responded to the questionnaire and those who did not, i.e. a risk for selective drop-out, which requires caution when interpreting the study results. Reasons for non-response are unknown. A possible reason could be that the interest in responding was low due to dissatisfaction [54]. The patients in the standard practice group were significantly older at baseline. Higher satisfaction scores from older patients have been reported in previous research [33, 34, 55] and therefore this factor cannot explain the differences in perceived care found in our study.

This study reports on patients' perceptions of quality of care in the short term. Patient satisfaction may change based on end result, however, considering that re-visits to the GP or orthopaedic consultation were very low, as presented previously [20], it could be argued that the results were maintained over time.

The fact that only one physiotherapist and one orthopaedic surgeon performed the assessments, as well as other limitations of the study, limits generalisability of the findings and the results must be interpreted with caution.

\section{Future research}

The aim for future research on physiotherapist-led orthopaedic triage should preferably focus on multicentre 
studies with several different physiotherapists and orthopaedic surgeons. While costs for physiotherapist are substantially lower than those for orthopaedic surgeons, cost effectiveness of this model should be further investigated. One problematic area is that the education as well as the professional role of physiotherapists differs internationally, which complicates generalisation. The most appropriate education for physiotherapists working in this role could be valuable to explore, and to consider for different healthcare settings.

\section{Conclusions}

This paper reports that patients in both groups perceived good quality of care, with patients in the physiotherapist-led triage perceiving significantly higher quality of care. Additionally, this model of care seems to meet patients' expectations and lead to a greater intention to follow advice. Our findings are in line with existing literature that this model of care provides an opportunity to shape patient-centred care that can improve access and offer care on the most appropriate level, with maintained good quality of care.

\section{Endnotes}

\section{${ }^{1} \mathrm{KUPP}$ is the Swedish name for QPP}

\begin{abstract}
Abbreviations
GP, General Practitioner; IO, Identity-orientated approach; MT, Medicaltechnical competence; NA, Not applicable; OMT, Orthopaedic Manipulative Therapy; PR, Perceived Reality; Q1; Q3, First quartile; third quartile; QPP, Quality from the Patient's Perspective; SD, Standard Deviation; SI, Subjective importance.
\end{abstract}

\section{Acknowledgements}

The authors gratefully acknowledge Maria Dottori for help with the data collection, and professor emerita Jane Carlsson for general support.

\section{Funding}

Obtained from the Department of Healthcare Services, Region Västra Götaland, Sweden.

\section{Availability of data and materials}

Participants did not consent to public release of their data. However, data can be made available on request for researchers who meet the criteria for access to confidential data, from Maria EH Larsson, Research and Development Primary Health Care, Region Västra Götaland, Kungsgatan 12, 6 th floor.

\section{Authors' contributions}

KSS participated in the design, performed the clinical assessment, analysis and interpretation of data, and manuscript writing. SB participated in the analysis and interpretation of data, and manuscript writing. MEHL participated in the design, analysis and interpretation of data as well as manuscript writing. All authors read and approved the final manuscript.

\section{Competing interest}

The authors declare that they have no competing interest.

\section{Ethics approval and consent to participate}

Upon inclusion patients gave verbal consent to participate and prior to the consultation they provided written informed consent. The study was approved by the Regional Ethical Review Board in Gothenburg, Sweden (09.09.09, Reference no: 382-09).

\section{Author details}

'Department of Health and Rehabilitation, Institute of Neuroscience and Physiology at Sahlgrenska Academy, University of Gothenburg, Box 430405 30 Gothenburg, Sweden. ${ }^{2}$ Närhälsan Tjörn Rehabilitation Clinic, Primary Health Care, Region Västra Götaland, Syster Ebbas väg 1, 47194 Kållekärr, Sweden. ${ }^{3}$ Närhalsan Research and Development Primary Health Care, Region Västra Götaland, Kungsgatan 12, 6th floor, 41118 Gothenburg, Sweden.

Received: 17 February 2016 Accepted: 2 June 2016

Published online: 10 June 2016

\section{References}

1. Gardiner J, Turner P. Accuracy of clinical diagnosis of internal derangement of the knee by extended scope physiotherapists and orthopaedic doctors: Retrospective audit. Physiotherapy. 2002;88(3):153-7.

2. Morris J, Grimmer-Somers K, Kumar S, Murphy K, Gilmore L, Ashman B, Perera C, Vine K, Coulter C. Effectiveness of a physiotherapy-initiated telephone triage of orthopedic waitlist patients. Patient Relat Outcome Meas. 2011;2:151-9.

3. Bath B, Janzen B. Patient and referring health care provider satisfaction with a physiotherapy spinal triage assessment service. J Multidiscip Healthc. 2012; 5:1-15.

4. Desmeules F, Toliopoulos P, Roy JS, Woodhouse LJ, Lacelle M, Leroux M, Girard S, Feldman DE, Fernandes JC. Validation of an advanced practice physiotherapy model of care in an orthopaedic outpatient clinic. BMC Musculoskelet Disord. 2013;14:162.

5. Daker-White G, Carr AJ, Harvey I, Woolhead G, Bannister G, Nelson I, Kammerling M. A randomised controlled trial. Shifting boundaries of doctors and physiotherapists in orthopaedic outpatient departments. J Epidemiol Community Health. 1999;53(10):643-50.

6. Robarts S, Kennedy D, MacLeod AM, Findlay H, Gollish J. A framework for the development and implementation of an advanced practice role for physiotherapists that improves access and quality of care for patients. Healthc Q. 2008;11(2):67-75.

7. Blackburn MS, Cowan SM, Cary B, Nall C. Physiotherapy-led triage clinic for low back pain. Aust Health Rev. 2009;33(4):663-70.

8. Razmjou H, Robarts S, Kennedy D, McKnight C, MacLeod AM, Holtby R. Evaluation of an advanced-practice physical therapist in a specialty shoulder clinic: Diagnostic agreement and effect on wait times. Physiother Can. 2013; 65(1):46-55

9. Morris J, Grimmer K, Gilmore L, Perera C, Waddington G, Kyle G, Ashman B, Murphy K. Principles to guide sustainable implementation of extendedscope-of-practice physiotherapy workforce redesign initiatives in Australia: Stakeholder perspectives, barriers, supports, and incentives. J Multidiscip Healthc. 2014;7:249-58.

10. Oldmeadow LB, Bedi HS, Burch HT, Smith JS, Leahy ES, Goldwasser M. Experienced physiotherapists as gatekeepers to hospital orthopaedic outpatient care. Med J Aust. 2007;186(12):625-8.

11. Morris JH, James RE, Davey R, Waddington G. What is orthopaedic triage? A systematic review. J Eval Clin Pract. 2014;21(1):128-36.

12. Maddison P, Jones J, Breslin A, Barton C, Fleur J, Lewis R, McSweeney L, Norgain C, Smith S, Thomas C, et al. Improved access and targeting of musculoskeletal services in northwest Wales: Targeted early access to musculoskeletal services (TEAMS) programme. Br Med J. 2004;329(7478): $1325-7$

13. Aiken $\mathrm{AB}, \mathrm{McC}$ oll MA. Diagnostic and treatment concordance between a physiotherapist and an orthopedic surgeon - A pilot study. J Interprof Care. 2008;22(3):253-61.

14. Dickens V, Ali F, Gent H, Rees A. Assessment and diagnosis of knee injuries: The value of an experienced physiotherapist. Physiotherapy. 2003;89(7):417-42.

15. Napier C, McCormack RG, Hunt MA, Brooks-Hill A. A physiotherapy triage service for orthopaedic surgery: An effective strategy for reducing wait times. Physiother Can. 2013;65(4):358-63.

16. Moore JH, Goss DL, Baxter RE, DeBerardino TM, Mansfield LT, Fellows DW, Taylor DC. Clinical diagnostic accuracy and magnetic resonance imaging of patients referred by physical therapists, orthopaedic surgeons, and nonorthopaedic providers. J Orthop Sports Phys Ther. 2005;35(2):67-71.

17. Mackay C, Davis AM, Mahomed N, Badley EM. Expanding roles in orthopaedic care: A comparison of physiotherapists and orthopaedic surgeon recommendations for triage. J Eval Clin Pract. 2009;15(1):178-83. 
18. Oakley C, Shacklady C. The Clinical Effectiveness of the Extended-Scope Physiotherapist Role in Musculoskeletal Triage: A Systematic Review. Musculoskeletal Care. 2015;13(4):204-21.

19. McEvoy C, Wiles L, Bernhardsson S, Grimmer K: Triage for Patients with Spinal Complaints: A Systematic Review of the Literature. Physiother Res Int. 2015; doi: 10.1002/pri.1639

20. Samsson K, Larsson MEH. Physiotherapy screening of patients referred for orthopaedic consultation in primary healthcare - A randomised controlled trial. Man Ther. 2014;19(5):386-91.

21. Samsson KS, Larsson MEH. Physiotherapy triage assessment of patients referred for orthopaedic consultation - Long-term follow-up of healthrelated quality of life, pain-related disability and sick leave. Man Ther. 2015; 20(1):38-45.

22. Hussenbux A, Morrissey D, Joseph C, McClellan CM. Intermediate Care pathways for musculoskeletal conditions - Are they working? A systematic review. Physiotherapy. 2015;101(1):13-24.

23. Stanhope J, Grimmer-Somers K, Milanese S, Kumar S, Morris J. Extended scope physiotherapy roles for orthopedic outpatients: an update systematic review of the literature. J Multidiscip Healthc. 2012;5:37-45.

24. Quality and Efficiency in Swedish Health Care. http://www.socialstyrelsen.se/lists/ artikelkatalog/attachments/18336/2011-5-18.pdf. Accessed on 22 August 2013.

25. Kitson A, Marshall A, Bassett K, Zeitz K. What are the core elements of patient-centred care? A narrative review and synthesis of the literature from health policy, medicine and nursing. J Adv Nurs. 2013;69(1):4-15.

26. Quality assurance standards of physiotherapy service delivery. http://www.csp. org.uk/publications/quality-assurance-standards. Accessed on 22 August 2013.

27. Kennedy DM, Robarts S, Woodhouse LJ. Patients are satisfied with advanced practice physiotherapists in a role traditionally performed by orthopaedic surgeons. Physiother Can. 2010;62:298-305.

28. Wilde B, Starrin B, Larsson G, Larsson M. Quality of Care from a Patient Perspective: A Grounded Theory Study. Scand J Caring Sci. 1993;7(2):113-20.

29. Moher D, Hopewell S, Schulz KF, Montori V, Gøtzsche PC, Devereaux PJ, Elbourne D, Egger M, Altman DG. CONSORT 2010 explanation and elaboration: updated guidelines for reporting parallel group randomised trials. BMJ. 2010;340:C869.

30. Wilde B, Larsson G, Larsson M, Starrin B. Quality of care: Development of a Patient-Centred Questionnaire based on a Grounded Theory Model. Scand J Caring Sci. 1994;8(1):39-48.

31. Larsson G, Wilde Larsson B, Munck I. Refinement of the questionnaire "Quality of Care From the Patient's Perspective" using structural equation modelling. Scand J Caring Sci. 1998;12(2):111-8.

32. Wilde Larsson $B$, Larsson $G$. Patients' views on quality of care and attitudes towards re-visiting providers. Int J Health Care Qual Assur. 2009;22(6):600-11.

33. Grøndahl VA, Karlsson I, Hall-Lord ML, Appelgren J, Wilde-Larsson B. Quality of care from patients' perspective: Impact of the combination of personrelated and external objective care conditions. J Clin Nurs. 2011;20(17-18): 2540-51.

34. Wilde Larsson B, Larsson G, Wickman Chantereau M, Staël von Holstein K. International comparisons of patients' views on quality of care. Int J Health Care Qual Assur. 2005;18(1):62-73.

35. Wilde Larsson B, Larsson G. Development of a short form of the Quality from the Patient's Perspective (QPP) questionnaire. J Clin Nurs. 2002;11(5):681-7.

36. Holter H, Sandin-Bojö AK, Gejervall AL, Wikland M, Wilde-Larsson B, Bergh C Quality of care in an IVF programme from a patient's perspective: Development of a validated instrument. Hum Reprod. 2014;29(3):534-47.

37. Wilde-Larsson B, Larsson G, Kvist LJ, Sandin-Bojö AK. Womens' opinions on intrapartal care: Development of a theory-based questionnaire. J Clin Nurs. 2010;19(11-12):1748-60.

38. Sandsdalen T, Rystedt I, GrøndahI VA, Hov R, Høye S, Wilde-Larsson B. Patients' perceptions of palliative care: Adaptation of the Quality from the Patient's Perspective instrument for use in palliative care, and description of patients' perceptions of care received Psychosocial. BMC Palliat Care. 2015;14(1):1.

39. Jangland $E$, Carlsson $M$, Lundgren $E$, Gunningberg $L$. The impact of an intervention to improve patient participation in a surgical care unit: A quasiexperimental study. Int J Nurs Stud. 2012;49(5):528-38.

40. Interference for Means: Comparing Two Independent Samples. http://www.stat.ubc.ca/ rollin/stats/ssize/n2.html. Accessed on 1 April 2016.

41. Reeve S, May S. Exploration of patients' perspectives of quality within an extended scope physiotherapists' spinal screening service. Physiother Theory Pract. 2009;25(8):533-43.
42. Verbeek J, Sengers MJ, Riemens L, Haafkens J. Patient expectations of treatment for back pain: A systematic review of qualitative and quantitative studies. Spine. 2004;29(20):2309-18.

43. Stenberg G, Fjellman-Wiklund A, Ahlgren C. "Getting confirmation": Gender in expectations and experiences of healthcare for neck or back patients. J Rehabil Med. 2012;44(2):163-71.

44. May SJ. Patient satisfaction with management of back pain. Part 1: What is satisfaction? Review of satisfaction with medical management. Physiotherapy. 2001;87(1):4-5.

45. Noble PC, Fuller-Lafreniere S, Meftah M, Dwyer MK: Challenges in Outcome Measurement: Discrepancies Between Patient and Provider Definitions of Success. Clin Orthop Relat Res. 2013;471(11):3437-45.

46. Muntlin $\AA$, Gunningberg L, Carlsson M. Patients' perceptions of quality of care at an emergency department and identification of areas for quality improvement. J Clin Nurs. 2006;15(8):1045-56.

47. Doyle C, Lennox L, Bell D: A systematic review of evidence on the links between patient experience and clinical safety and effectiveness. BMJ Open. 2013;3(1). doi: 10.1136/bmjopen-2012-001570.

48. Barbosa CD, Balp MM, Kulich K, Germain N, Rofail D. A literature review to explore the link between treatment satisfaction and adherence, compliance, and persistence. Patient Prefer Adherence. 2012;6:39-48.

49. Richardson B, Shepstone L, Poland F, Mugford M, Finlayson B, Clemence N. Randomised controlled trial and cost consequences study comparing initial physiotherapy assessment and management with routine practice for selected patients in an accident and emergency department of an acute hospital. Emerg Med J. 2005;22(2):87-92.

50. Ball STE, Walton K, Hawes S. Do emergency department physiotherapy practitioner's, emergency nurse practitioners and doctors investigate, treat and refer patients with closed musculoskeletal injuries differently? Emerg Med J. 2007;24(3):185-8

51. Sitzia J, Wood N. Response rate in patient satisfaction research: An analysis of 210 published studies. Int J Qual Health Care. 1998;10(4):311-7.

52. Hush JM, Cameron K, Mackey M. Patient satisfaction with musculoskeletal physical therapy care: A systematic review. Phys Ther. 2011;91(1):25-36.

53. Jakobsson L, Holmberg L. Quality from the patient's perspective: A one-year trial. Int J Health Care Qual Assur. 2012;25(3):177-88.

54. Kinnersley P, Stott N, Peters T, Harvey I, Hackett P. A comparison of methods for measuring patient satisfaction with consultations in primary care. Fam Pract. 1996;13(1):41-51.

55. Fröjd C, Leo Swenne C, Rubertsson C, Gunningberg L, Wadensten B. Patient information and participation still in need of improvement: evaluation of patients' perceptions of quality of care. J Nurs Manag. 2011;19(2):226-36.

\section{Submit your next manuscript to BioMed Central and we will help you at every step:}

- We accept pre-submission inquiries

- Our selector tool helps you to find the most relevant journal

- We provide round the clock customer support

- Convenient online submission

- Thorough peer review

- Inclusion in PubMed and all major indexing services

- Maximum visibility for your research

Submit your manuscript at www.biomedcentral.com/submit 